\title{
Bifurcation analysis of a host-parasitoid ecological model with the beddington-deangelis functional response
}

\author{
Tanuja Agrawal \\ Department of Applied Mathematics, Z.H. College of Engineering and Technology, AMU, Aligarh, UP 202 002, India \\ E-mail: tanu.18aug@gmail.com
}

Copyright $\odot 2014$ Tanuja Agrawal. This is an open access article distributed under the Creative Commons Attribution License, which permits unrestricted use, distribution, and reproduction in any medium, provided the original work is properly cited.

\begin{abstract}
In this paper, a two species host-parasitoid model system is considered. The global dynamic behavior of the model is investigated through (local) stability results for its equilibriums and large time computer simulations. Many forms of complex dynamics such as chaos, periodic windows etc. are observed. The Hopf point and attractor crises exist for different set of parameter values.
\end{abstract}

Keywords: Predator-Prey; Bifurcation; Chaos; Stability.

\section{Introduction}

Hosts and parasitoids are mostly univoltine and have no overlap between successive generations. Therefore, their interactions can be modeled by discrete differences [15-18]. In 1929, Thompson [1] introduced a host-parasitoid model with a set of biological assumption. In 1935, Nicholson and Bailey [2] modified the Thompson's model [1] for better prediction of real life situations. After that, Rogers [3-4] applied the model of Holling to host-parasitoid system by assuming two kinds of limitation on Thompson's model and Nicholson-Bailey's model [2]. Many researchers [5], [10] produced many discrete type host-parasitoid models with different ecological factors. Tang and Chen [11] modified the Nicholson-Baley's model [2, 12] by introducing Holling type-II and type-III interactions.

Competition is an interaction between organisms or species in ecology. Competition is one of many biotic and abiotic factors that affect community structure due to limited resource (food, space and water). Competition among members of same species for the same resources in an ecosystem is known as intraspecific competition. Competition is not straightforward, and can occur in both direct and indirect fashion [13]. May [14] demonstrate that several discrete-time models describing the dynamics of isolated single species populations displayed chaos for large range of parameters? It has been very much in vogue for researchers to investigate the possible existence of bifurcations and chaos in ecological non-linear systems, much of which has been prompted by new and potential applications in different fields [9, 19]. In Holling response, it is assumed that predators do not interfere with one another's activities; thus competition among predators for food occurs only in case of depletion of prey.

Tang and Chen [11] reported the dynamic complexities of host-parasitoid interactions with a Holling type II functional response, and showed that discrete time host parasitoid models can produce much richer set of dynamic patterns than those of continuous models. Tang and Chen model [11] is

$$
\begin{aligned}
& H(t+1)=H(t) \exp \left[r\left(1-H(t) / k-\frac{a T P(t)}{\left(1+a T_{h} H(t)\right)}\right)\right] \\
& P(t+1)=H(t)\left[1-\exp \left(\frac{a T P(t)}{\left(1+a T_{h} H(t)\right)}\right)\right]
\end{aligned}
$$

Where

$H(t)$ is the host population size at generation $t$ 
$P(t)$ is the parasitoid population size at generation $t$

$r$ is the intrinsic growth rate

$k$ is the carrying capacity of the environment

$a$ is the instantaneous search rate

$T$ is the total time initially available for the search

$T_{h}$ is the handling time

In the present work, we study Tang and Chen model [11] with Beddington -DeAngelis functional response which is an extension of the prey-dependent Holling's type II functional response.

\section{Model}

One of well-known functional response is the Beddington De-Angelis functional response introduced by Beddington and De-Angelis et al. independently. The main difference of this functional response from classical Holling type response is that it contains an extra term presenting mutual interference by predators. Introducing Beddington DeAngelis functional response into Tang and Chen model [11], model becomes

$$
\begin{aligned}
& H(t+1)=H(t) \exp \left[r(1-H(t) / k)-\frac{a T P(t)}{1+a T_{h} H(t)+b P(t)}\right] \\
& P(t+1)=H(t)\left[1-\exp \left(-\frac{a T P(t)}{1+a T_{h} H(t)+b P(t)}\right)\right]
\end{aligned}
$$

Where all the variables and parameters are the same as defined in (1.1).

\section{Stability analysis}

In this section, the existence and local stability of the non-negative equilibrium points of system (1.2) is investigated. System (1.2) has two non-negative equilibrium points namely the total extinction solution $E_{0}=(0,0)$ and the coexistence solution for the two species $E_{1}=\left(H^{*}, P^{*}\right)$. The equilibrium point $E_{1}=\left(H^{*}, P^{*}\right)$ satisfies the following equations:

$$
\begin{aligned}
& H^{*}=\frac{\ln q\left[1+2 k\left(1-\frac{\ln q}{r}\right)\right]}{a(1+q)} \\
& P^{*}=k\left(1-\frac{\ln q}{r}\right)(1+q) \\
& r\left(1-\frac{H^{*}}{k}\right)
\end{aligned}
$$

Note that the equilibrium point $E_{1}=\left(H^{*}, P^{*}\right)$ cannot be solved in a closed form.

After obtaining equilibrium points, we need to check what happens to the dynamic variables $H$ and $P$ when an equilibrium point is slightly perturbed. Such knowledge is obtained by calculating the Jacobian matrix. System (1.2) can be rewritten in the form $H(t+1)=F(H(t), P(t))$

$$
P(t+1)=G(H(t), P(t))
$$

For which the Jacobian matrix is

$$
\left(\begin{array}{ll}
\frac{\partial F}{\partial H} & \frac{\partial F}{\partial P} \\
\frac{\partial G}{\partial H} & \frac{\partial G}{\partial P}
\end{array}\right)
$$

The Jacobian matrix of system (1.2) at the equilibrium point $E_{0}=(0,0)$ is

$$
J(0,0)=\left(\begin{array}{cc}
e^{r} & 0 \\
0 & 0
\end{array}\right)
$$


Accordingly, the eigenvalues are $\lambda_{1}=e^{r}, \lambda_{2}=0$. From here, it can be concluded that $E_{0}=(0,0)$ is an unstable $\left(\left|\lambda_{1}\right|>1\right)$.

\section{Bifurcation analysis}

It is very much clear from the previous section that system (1.2) cannot be solved explicitly. Thus we have to study the long term behavior of this system by numerical simulation. Fixing $r$ between 0 and 3.5 we have different bifurcation diagrams for host and parasitoid populations. Here we have shown the comparison between the bifurcation diagrams of model (1.1) and (1.2).

Figure 1 shows the bifurcation diagram of host and parasitoid population for $r$ varying between 0 and 3.5 for Holing type II functional response:

(a)

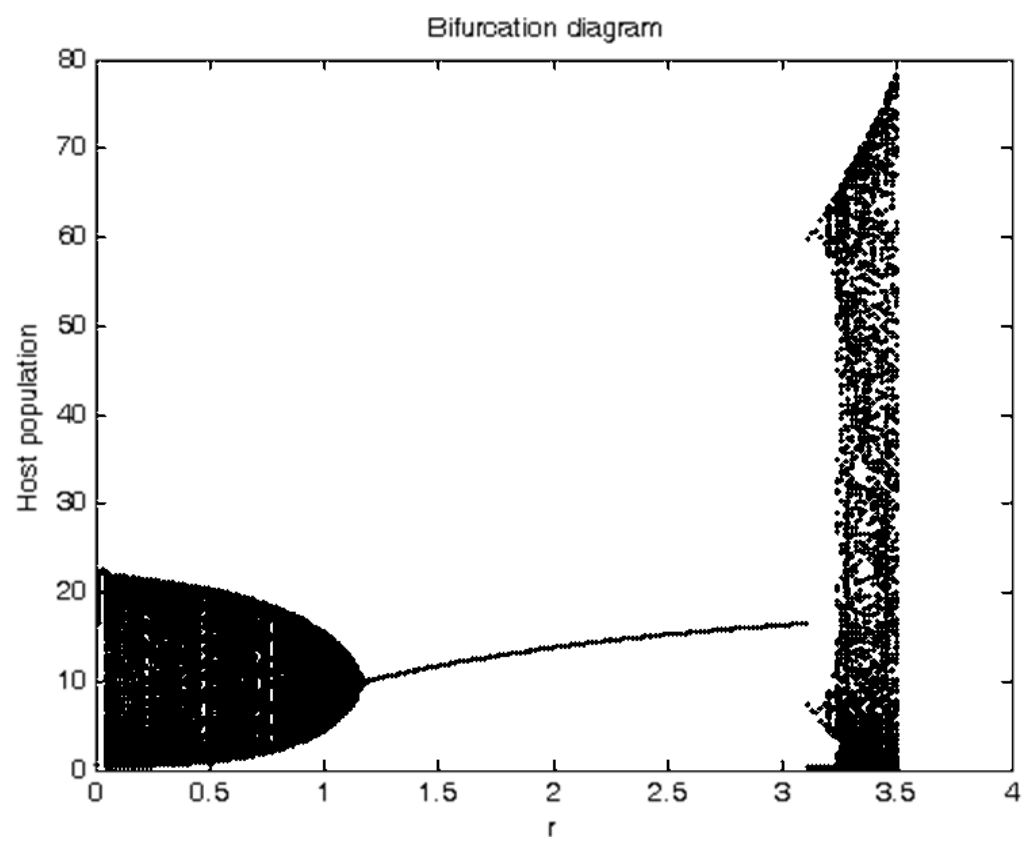

(b)

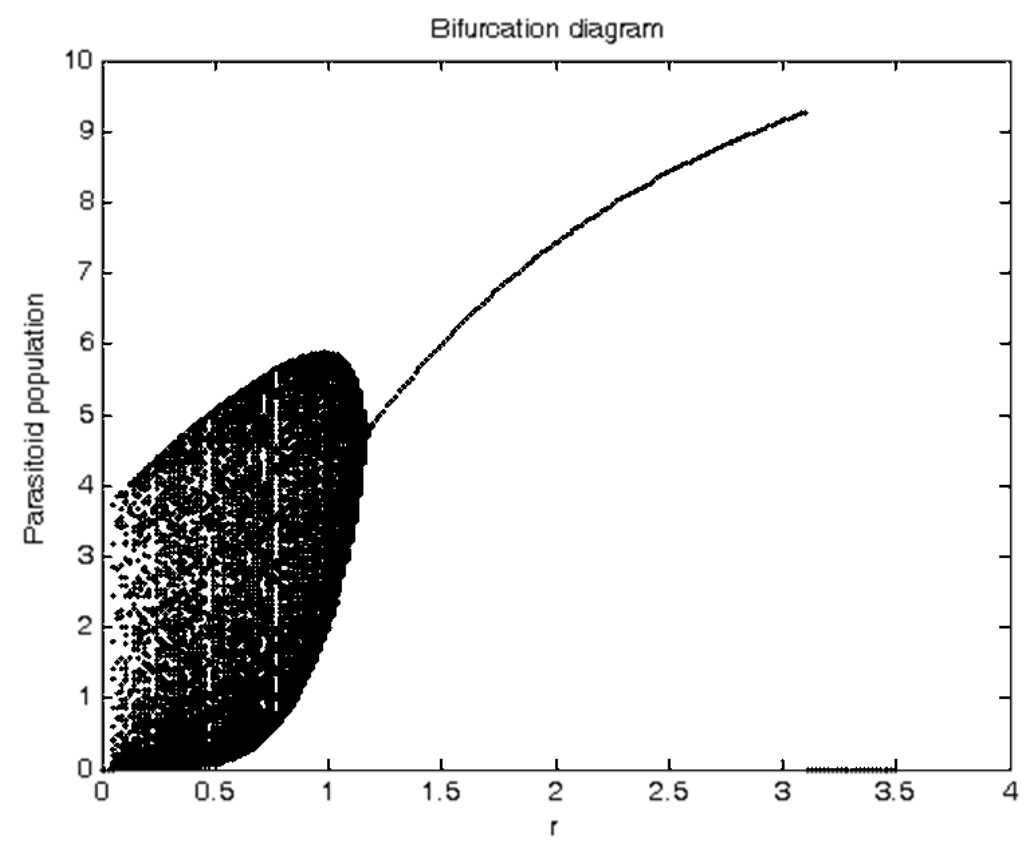

Fig. 1: (A) Bifurcation Diagram of Host Population (B) Parasitoid Population for $a=1.5, T=0.5, T_{h}=0.3, k=22.47$. 
Figure 2 shows the bifurcation diagram of host and parasitoid population for $r$ varying between 0 and 3.5 for Bedding ton De-Angelis functional response.

(a)

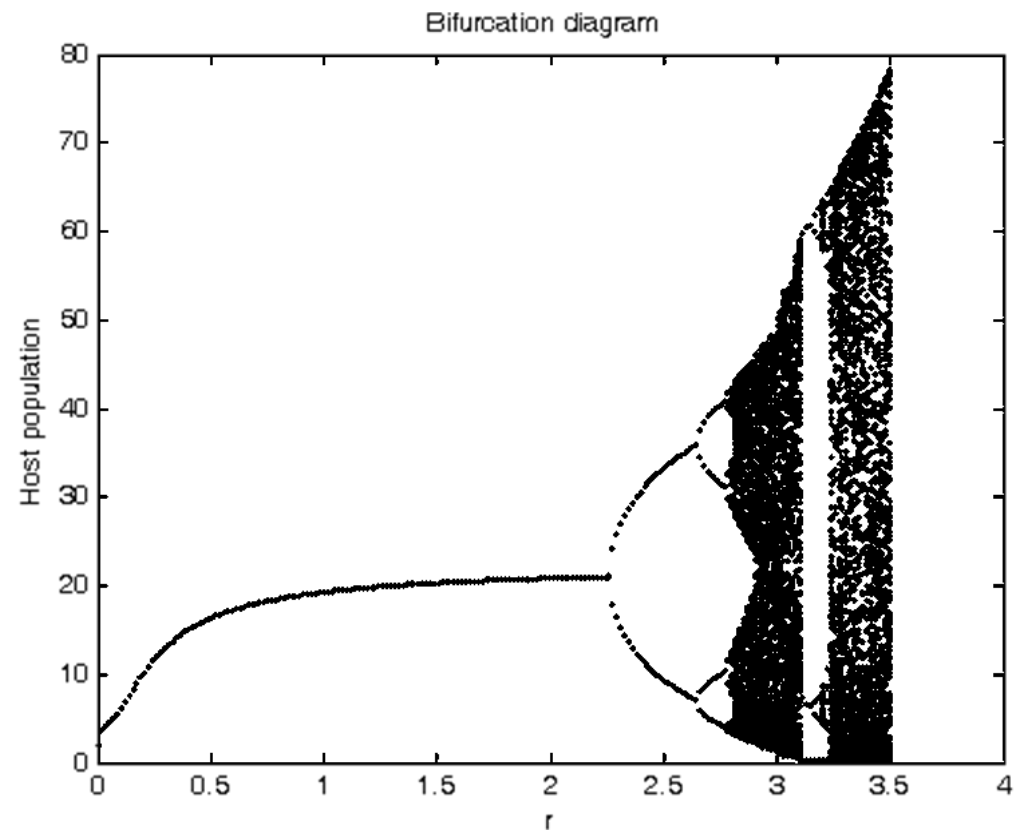

(b)

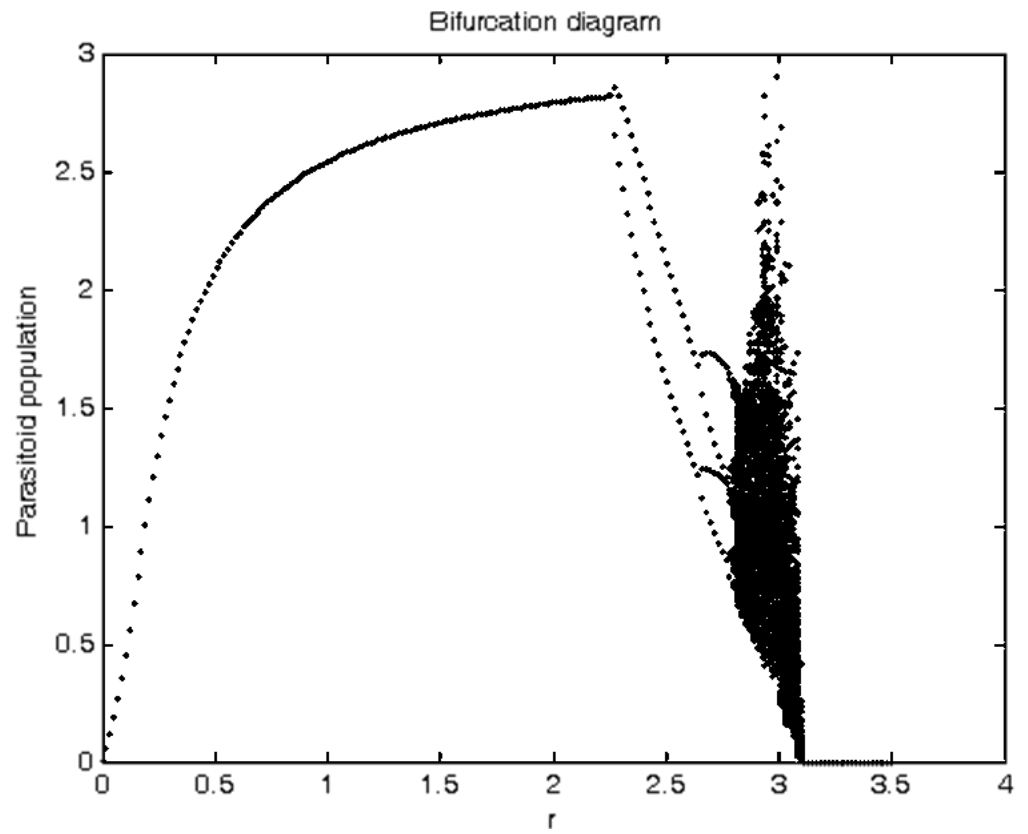

Fig. 2: (A) Bifurcation Diagram of Host Population (B) Parasitoid Population for $a=1.5, T=0.5, T_{h}=0.3, b=1.5, k=22.47$.

\section{Conclusion}

A Host-parasitoid model with intraspecific competition is proposed and analyzed. We have done the bifurcation analysis with respect to instantaneous search rate for different types of intraspecific competitions taking as parameter separately. We observe Hopf bifurcation and attractor crises. Stable and oscillatory coexistence of host and parasitoid are observed in different types of competitions for some values of instantaneous search rate. We have given the stability analysis of model (1.2) using Bedding ton functional response and then the bifurcation analysis through computer simulations. We have shown the bifurcation diagram of host and parasitoid population for model (1.1) and (1.2) for different values of $a, T, T_{h}, b$ and fixing the range of $r$ between 0 and 3.5. Identifying complicated, possibly chaotic, dynamics in population data has remained a major challenge in ecological studies [20] and it is not easier to predict this 
complexity by the theory. The present research helps to understand the dynamical behavior of host-parasitoid interactions with intraspecific competition which can be used to improve the classical biological control of pests.

\section{References}

[1] W.R. Thompson, On the relative value of parasites and predators in the biological control of insect pests, Bull Entomol Res 19 (1929) $343-$ 350 .

[2] V.A. Bailey, A.J. Nicholson and E.J. Williams, Interaction between hosts and parasites when some host individuals are more difficult to find than others, J Theor Biol. 3 (1962) 1-18.

[3] D.J. Rogers, Random search and insect population models, J Animal Ecol. 41 (1972)369-383.

[4] M.P. Hassell, D.J. Rogers, Insect Parasite Response in the Development ofPopulation Model. Journal of Animal Ecology 41 (1972)661-676.

[5] S.R.J. Jang, Discrete-time host parasitoid models with Allee effects: Densitydependence versus parasitism. Journal of Difference Equations and Applications 17(2011) 525-539.

[6] J.R. Beddington, C.A. Free, J.H. Lawton, Dynamic complexity in predator-preymodels framed in difference equations, Nature 225 (1975) 58 60 .

[7] R.M. May, Limit cycles in predator-prey communities, Science 177 (1972) 900-902.

[8] Y. Xiao, S. Tang S, The effect of initial density and parasitoid intergenerational survival rate on classical biological control, Chaos, Solitons and Fractals 37 (2008) 1048-1058.

[9] C. Xu. M.S. Boyce, Dynamic complexities in a mutual interference hostparasitoid model, Chaos, Solitons and Fractals 24 (2005) 175-182.

[10] L. Zhu, M. Zhao, Dynamic complexity of host-parasitoid ecological modelwith the Hassell growth function for the host, Chaos, Solitons and Fractals, 39 (2009) 1259-1269.

[11] S. Tang, L. Chen, Chaos in functional response host-parasitoid ecosystemModels, Chaos, Solitons and Fractals, 13 (2002) 875-884.

[12] A.J. Nicholson, V.A. Bailey, The balance of animal populations, Part1.ProcZoolSocLondan, (1935) 551-598

[13] S. Sahney, M.J. Benton, P.A. Ferry, Links between global taxonomic diversity, ecological diversity and the expansion of vertebrates on land, Biology Letters 6 (2010) 544-547.

[14] R. M. May, Simple mathematical models with very complicated dynamics.Nature1976; 261:459-67.

[15] M. P. Hassell, The dynamics of arthropod predator-prey systems. Princeton: University Press Princeton NJ; 1978.

[16] S. R. Jang, S. L. Diamond, A host-parasitoid interaction with Allee effects on the host. Comput Math Appl 2007; 53:89-103.

[17] R. Kon, Y. Takeuchi, Permanence of host-parasitoid systems. Nonlinear Anal 2001; 47:1383-93.

[18] E. G. Gu, The nonlinear analysis on a discrete host-parasitoid model with pesticidal interference. Commun Nonlinear Sci Numer Simul 2009; 14:2720-7.

[19] Y. N. Xiao, D. Z. Cheng, S. Y. Tang, Dynamic complexity in predator prey ecosystem models with age structure for predators. Chaos, Solitons and fractals 2002; 14:1403-11.

[20] G. Sugihara,R. M. May, Nonlinear forecasting as a way of distinguishingchaos from measurement error in time series, Nature 344 (1990) $734-$ 741. 